\title{
Downregulated MicroRNA-195 in
} the Bicuspid Aortic Valve Promotes Calcification of Valve Interstitial Cells via Targeting SMAD7

\author{
Junjie Du ${ }^{a} \quad$ Rui Zheng ${ }^{a} \quad$ Feng Xiao $^{b} \quad$ Shuai Zhanga Keshuai He ${ }^{a} \quad$ Junjie Zhang ${ }^{a}$ \\ Yongfeng Shao ${ }^{a}$
}

Department of Cardiovascular Surgery, the First Affiliated Hospital of Nanjing Medical University, Nanjing, 'Department of Cardiology, Wuxi People's Hospital Affiliated to Nanjing Medical University, Wuxi, China

\section{Key Words}

Bicuspid aortic valve • Valvular calcification • Valve interstitial cell • MicroRNA-195 • SMAD7

\begin{abstract}
Background/Aims: Aortic stenosis caused by leaflet calcification in the bicuspid aortic valve (BAV) is more accelerative than that in the tricuspid aortic valve (TAV). MicroRNA-195 (miR-195) is downregulated more in stenotic than in insufficient BAVs, but its expression in BAVs compared with TAVs is unclear. We aimed to investigate the roles of miR-195 and its calcification-related target SMAD7 in stenotic BAVs compared with those in TAVs. Methods: Twenty-one stenotic BAV and 29 TAV samples were collected from surgical patients and examined for the expression of miR-195 and SMAD7 by RT-PCR. The samples were also assessed by western blotting and immunohistochemistry for the functional protein alteration associated with calcification. Dual-luciferase assay was performed to determine the putative target of miR-195 before the effects of miR-195 expression on osteogenic progression was demonstrated in cultured porcine valve interstitial cells (VICs). Results: Compared with TAV, the expression of miR-195 was remarkably lower in the BAV leaflet with higher expression of SMAD7, which was then validated as a direct target of miR-195. Their negative correlation was then confirmed in cultured VICs. Under an osteogenic environment, the cellular calcification was promoted in miR-195-repressed VICs expressing higher BMP-2 and Runx2 and higher activity of MMP-2 compared with the controls. Finally, higher MMP-2 and MMP-9 expression and more collagen distribution were observed in BAV than TAV samples. Conclusions: miR195 is downregulated more in stenotic BAV than TAV in this study. The downregulation of miR-195 is associated with valvular calcification via targeting SMAD7, which promotes the remodeling of the extracellular matrix.

J. Du and R. Zheng contributed equally to this article.




\section{Introduction}

Bicuspid aortic valve (BAV) disease is one of the most common congenital anomalies of the human heart, present in 1-2\% of the population [1]. Aortic stenosis (AS) is the most typical form of symptomatic BAV disease and has a significant clinical impact on BAV patients [2]. In adults, the development of AS in BAV is often due to leaflet calcification, which occurs in a similar fashion to that seen in patients with tricuspid aortic valve (TAV) calcification but in a more accelerated progression [3]. Therefore, it is essential to explore the unique mechanisms of leaflet calcification in BAV patients to better understand this important disease.

It is well known that the key component of valvular calcification development involves the reprogramming and transdifferentiation of valve interstitial cells (VICs) to osteoblastlike cells by expressing osteogenic signals, notably the bone morphogenetic proteins (BMPs) of the transforming growth factor- $\beta$ (TGF- $\beta$ ) family [4-6]. Of the BMP family, BMP-2, with its downstream element, the transcription factor runt-related transcription factor 2 (RunX2), is a potent stimulant of valvular calcification in vivo and in vitro [7]. However, the mechanisms by which VICs form calcified aggregates and, more importantly, the factors that regulate these processes are still not well defined.

Recent advances have identified microRNAs (miRs) as key regulators that manipulate several key checkpoints in the cellular processes involved in vascular calcification $[8,9]$. Furthermore, accumulating evidence has proven that miRs also mediate the progress of osteoblastic differentiation in VICs [10-12]. Recently, published microRNA profiling data of human BAV tissues have identified several altered miRs, including miR-26a, miR-30b, and miR-195, which may participate in the progress of valvular calcification [13]. While valvular calcification in BAV has been traditionally linked to genetic predisposition in relevant cells, hemodynamic abnormalities are increasingly indicated as potential pathogenic contributors. Casey and colleagues recently showed that disturbed flow present on the fibrosa side of the aortic valve stimulates endothelial cells to regulate shear-responsive miRNAs, such as miR486, miR-139, and miR-192, to induce aortic valve disease progression [14]. Therefore, it is understandable that BAV patients might have earlier diseased valves given their congenital deformation and poorer hemodynamic condition since birth. However, the exact roles and mechanisms of these miRs in valvular calcification in BAV have not been well studied yet.

There are many reasons to perform aortic valve replacement in clinical practice. The leading cause is symptomatic valve stenosis mainly due to degenerative valves in Western countries and rheumatic valves in developing countries such as China. In both cases, the presence of calcification in the aortic valve is responsible for valve stenosis. BAV patients with symptoms of valve stenosis, on the other hand, represent a special population that needs the same surgical treatment. Therefore, in the present study, we aimed to investigate the roles of miRs in the regulation of valvular calcification in calcified BAVs compared with those in stenotic TAVs by screening several miRs in human calcified BAV leaflets and in TAV samples from patients with either rheumatic or degenerative valve diseases. We then investigated the potential signaling pathway associated with the downregulated miR-195 in BAVs and their calcification-related target gene SMAD7.

\section{Materials and Methods}

\section{Study Population and Aortic Valve Collection}

The aortic valve leaflets used in the experiments were collected from patients who underwent aortic valve replacement at our institution. The institutional ethics committee of the First Affiliated Hospital of Nanjing Medical University approved the study, and all the patients provided written informed consent. Preoperatively, the functional state of the aortic valve was determined by echocardiography. The leaflets were inspected at the time of surgery for morphology. All BAV samples $(n=21)$ examined for this study had type 1 bicuspid aortic valve leaflets, with fusion of the right and left coronary leaflets. The TAVs were separated into two groups: rheumatic aortic valve group (RTV, $n=15)$ and degenerative aortic valve group ( $D T V, n=14)$, 


\begin{tabular}{|c|c|c|}
\hline Jar Dhycinlnay & Cell Physiol Biochem 2017;4 & 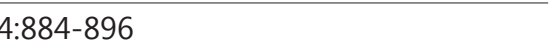 \\
\hline and Biochemistry & $\begin{array}{l}\text { DOI: } 10.1159 / 000485356 \\
\text { Published onlline: November 24, } 2017\end{array}$ & $\begin{array}{l}\text { O } 2017 \text { The Author(s). Published by S. Karger AG, Basel } \\
\text { www.karger.com/cpb }\end{array}$ \\
\hline
\end{tabular}

Table 1. Patient demographics and clinical characteristics

\begin{tabular}{|c|c|c|c|c|c|}
\hline \multirow[b]{2}{*}{ Variable } & \multirow{2}{*}{$\begin{array}{l}\mathrm{BAV} \\
(\mathrm{n}=21)\end{array}$} & \multirow{2}{*}{$\begin{array}{c}\text { RTV } \\
(n=15)\end{array}$} & \multirow{2}{*}{$\begin{array}{c}\text { DTV } \\
(n=14)\end{array}$} & \multicolumn{2}{|c|}{$\mathrm{P}$ value } \\
\hline & & & & $\begin{array}{l}\text { BAV vs. } \\
\text { RTV }\end{array}$ & $\begin{array}{l}\text { BAV vs. } \\
\text { DTV }\end{array}$ \\
\hline Age in years & $\begin{array}{c}53.2 \pm \\
10.9\end{array}$ & $50.3 \pm 9.5$ & $62.2 \pm 7.6$ & 0.43 & 0.012 \\
\hline Women, \% (n) & $28.6(6)$ & $40(6)$ & $21.4(3)$ & 0.09 & 0.712 \\
\hline \multicolumn{6}{|l|}{ Risk factors } \\
\hline Hypertension, \% (n) & $38.1(8)$ & $6.7(1)$ & $35.7(5)$ & 0.051 & 1.000 \\
\hline Hypercholesterolemia, \% (n) & $14.3(3)$ & $6.7(1)$ & $42.9(6)$ & 0.626 & 0.112 \\
\hline Diabetes, \% (n) & $9.5(2)$ & $0(0)$ & $21.4(3)$ & 0.500 & 0.369 \\
\hline Smoking history, \% (n) & $42.9(9)$ & $20.0(3)$ & $35.7(5)$ & 0.282 & 0.737 \\
\hline $\mathrm{CKD}, \%(\mathrm{n})$ & $0(0)$ & $0(0)$ & $0(0)$ & $\mathrm{N} / \mathrm{A}$ & $\mathrm{N} / \mathrm{A}$ \\
\hline \multicolumn{6}{|l|}{ Echocardiographic parameters } \\
\hline LVEF, \% & $61.1 \pm 9.1$ & $61.4 \pm 5.2$ & $65.4 \pm 3.4$ & 0.874 & 0.056 \\
\hline Aortic valve area, $\mathrm{cm}^{2}$ & $\begin{array}{c}0.68 \pm \\
0.22\end{array}$ & $\begin{array}{c}0.75 \pm \\
0.28\end{array}$ & $\begin{array}{c}0.67 \pm \\
0.34\end{array}$ & 0.261 & 0.751 \\
\hline Mean gradient, mmHg & $\begin{array}{c}58.8 \pm \\
31.6\end{array}$ & $\begin{array}{c}45.1 \pm \\
20.7\end{array}$ & $\begin{array}{c}59.7 \pm \\
35.5\end{array}$ & 0.148 & 0.939 \\
\hline LVPW, mm & $11.5 \pm 1.9$ & $9.8 \pm 1.8$ & $11.8 \pm 2.4$ & 0.008 & 0.676 \\
\hline $\begin{array}{l}\text { Ascending aorta maximal diameter, } \\
\mathrm{mm}\end{array}$ & $40.3 \pm 7.7$ & $33.7 \pm 5.7$ & $36.2 \pm 7.1$ & 0.008 & 0.126 \\
\hline
\end{tabular}

according to the echocardiographic results and intrasurgical inspections. Tissue samples from each patient were sectioned into two equal parts. One part was flash frozen in liquid nitrogen and then stored for RNA or protein extraction; the other part was fixed in $10 \%$ formalin and embedded in paraffin.
Table 2. Sequences of primers for RT-PCR

\begin{tabular}{lcc}
\hline Name & Direction & Sequence \\
\hline \multirow{2}{*}{ U6 } & forward & 5'-GTGGGGAGAAGAGGACAGGAC-3' \\
& reverse & 5'-GTGGTACCCACTTTCGCACA-3' \\
hsa-SMAD7 & forward & 5'-TTCCTCCGCTGAAACAGGG -3' \\
& Reverse & 5'-CCTCCCAGTATGCCACCAC-3' \\
\multirow{2}{*}{ ssc-SMAD7 } & forward & 5'-CCAGAACCTATTTCCCATGC-3' \\
& Reverse & 5'-GGCACTGGGTGAGCAATACT-3' \\
\multirow{2}{*}{ hsa-GAPDH } & forward & 5'-AGAAGGCTGGGGCTCATTTG-3' \\
& Reverse & 5'-AGGGGCCATCCACAGTCTTC-3' \\
\multirow{2}{*}{ ssc-GAPDH } & forward & 5'-CCTCAACGACCACTTCGTCA-3' \\
& Reverse & 5'-TGTGTTGGGGGATCGAGTTG-3' \\
\hline
\end{tabular}

Real-Time Polymerase Chain Reaction for the Detection of miRs and SMAD7 Expression

Human valve leaflet samples and the porcine VICs transfected using the methods described in the following paragraph were used to extract miRs and mRNAs using the miRNeasy Mini Kit (Qiagen, German-

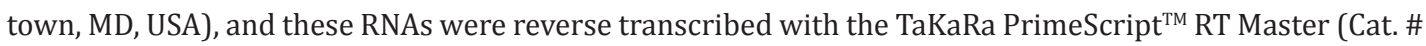
RR037A) according to the manufacturer's instructions.

Real-time polymerase chain reaction (RT-PCR) was performed using the ABI Real-Time Polymerase Chain Reaction System (7900HT Fast Real-Time PCR systems) and TaKaRa SYBR Premix Ex Taq ${ }^{\text {TM }}$ II (Cat. \# 820A) combined with either miR primers (Bulge-Loop ${ }^{\mathrm{TM}}$ miRNA qRT-PCR Primer Set, Rib Biological Technology Corporation, Guangzhou, China) or SMAD7 primers (Table 1). Sequences of primers for RT-PCR are listed in Table 2.

\section{Histologic Examination and Immunohistochemistry}

Formalin-fixed and paraffin-embedded tissues were used for Von Kossa and Sirius Red staining using semi-quantificational analysis. Von Kossa staining was carried out to reveal calcium deposition using a kit following manufacturer's instructions (24633, Polysciences). Sirius Red staining was used to examine collagen utilizing the Picro-Sirius Red Stain Kit (ab150681, Abcam). The tissues were also used for immunohistochemical staining for SMAD7, MMP-2 and MMP-9. Briefly, the sections were washed in Tris-buffered saline/0.1\% Tween-20 and were blocked in 5\% milk. The sections were incubated in primary antibodies $(1: 5,000)$ overnight, washed, and then incubated in biotinylated secondary antibodies (1:10, 000 ) for 30 minutes. The sections were treated with Vectastain ${ }^{\circledR}$ ABC reagent (Vector, Burlingame, CA, USA), followed by DAB treatment and counterstaining with hematoxylin. Software Image-Pro plus was used for signal quantitation. All the antibodies were from Abcam, MA, USA. 
Dual-luciferase Reporter As-

say

The pmirGLO dual-luciferase miRNA target expression vector (pmirGLO) containing both the firefly luciferase gene and Renilla luciferase gene was purchased from Promega (Madison, WI, USA). The 3'UTR segments of human SMAD7 mRNA (56-62 nt, GenBank accession no. NG_023330) containing the putative miR-195 binding sequences were amplified by RT-PCR and were inserted into the 3'-UTR downstream of the pmirGLO vector using the XbaI and SacI restriction sites and was named pmirGLO-UTR-WT. A site-directed gene mutagenesis kit (QuikChange XL, Agilent Technologies) was used to construct the mutant type of vector according to the manufacturer's protocol. The mutant construct was named pmirGLO-UTR-MUT, which carries a point mutation-i.e., a cytosine was replaced by a guanine in the 3'-UTR segments of human SMAD7 mRNA being complementary to the seed sequence of miR-195, as shown in Fig. 3b. Both constructs were confirmed by restriction enzyme digestion and sequencing (Invitrogen). Transient transfections were performed using Lipofectamine $^{\mathrm{TM}}$ (Invitrogen) as recommended by the manufacturer. Luciferase activities were measured at 48 hours after transfection using the Dual-Glo luciferase assay system (Promega), and firefly luciferase activities were normalized to Renilla luciferase activities. Experiments were performed in triplicate and were independently repeated three times. Sequences of primers for this assay are listed in Table 3.

\section{Cell Culture and Transient Transfection}

HEK293T cells were purchased from the Chinese Science Institute (Shanghai, China). The cells were seeded in 12-well plates in Dulbecco Modified Eagle Medium (Invitrogen) supplemented with 10\% fetal bovine serum, 1\% nonessential amino acids, L-glutamine, and penicillin $(100 \mathrm{U} / \mathrm{ml})$ with streptomycin $(100 \mu \mathrm{g} / \mathrm{ml})$ at $37^{\circ} \mathrm{C}$ under an atmosphere with $5 \%$ carbon dioxide. Cells in the logarithmic growth phase ( $\sim 80 \%$ confluence) were used for the dual-luciferase reporter assay.

Aortic VICs were harvested from 8 porcine (4-6 months old) hearts. Briefly, aortic valves were excised taking care not to introduce any non-leaflet tissue. The leaflets were gently scraped on the aortic and ventricular aspects to remove the endothelial layer, and the tissues were then sectioned into pieces approximately $2 \times 2 \mathrm{~mm}$ and then were explant cultured in growth medium (DMEM basic with penicillin G, streptomycin, amphotericin B, and 10\% fetal bovine serum) in an incubator supplied with $5 \%$ carbon dioxide for 10 days. The cells growing from the explants were subcultured, and the cells from passages 3 to 4 were used for additional studies.

Monolayers of VICs $\left(7.5 \times 10^{4}\right.$ cells $\left./ \mathrm{cm}^{2}\right)$ were transfected with $50 \mu \mathrm{M}$ of the miR-195 mimic or miR-195 inhibitor or a negative control (micrON ${ }^{\mathrm{TM}}$ miRNA mimic/inhibitor/nc_Standard, Ribobio Co., Guangzhou, China) using the Lipofectamine 3000 Transfection Kit (Thermo Fisher Scientific, Carlsbad, Canada) according to the manufacturer's recommendations. The transfected cells were further cultured with growth medium (DMEM with $10 \%$ fetal bovine serum) for 24 hours.

To study TGF- $\beta$ stimulated osteogenic progression in VICs, transfected cells were further cultured with or without $10 \mathrm{ng} / \mathrm{mL}$ TGF- $\beta$ (Sigma-Aldrich, St. Louis, MO, USA) for up to 14 days with $1 \%$ fetal bovine serum in the medium. The medium was changed every 3 days. The cells were harvested, and the total protein and RNA were extracted for analysis.

\section{Western Blot Analysis}

Western blot analysis was performed as follows. Cell lysates were prepared with a sample buffer $(100$ mmol/L Tris-HCl, pH 6.8, 2\% SDS, 0.02\% bromophenol blue, and 10\% glycerol). Equal amounts of protein per lane from different samples were loaded, and á-tubulin in the same sample was used as a loading control. After transfer to polyvinylidene difluoride membranes and blocking with 5\% skim milk solution, the protein in the membranes were incubated with primary antibodies followed by incubation with peroxidase-linked secondary antibodies specific to the primary antibodies. The blots were then developed using the ECL reagent (Amersham Biosciences), and images were captured using an imaging system (UVP BioImager) and were quantified. The images were captured digitally using a BioSpectrum AC imaging system, and the optical densities of the protein bands were normalized to the loading control bands and quantified. 


\section{Cellular Physiology Cell Physiol Biochem 2017;44:884-896

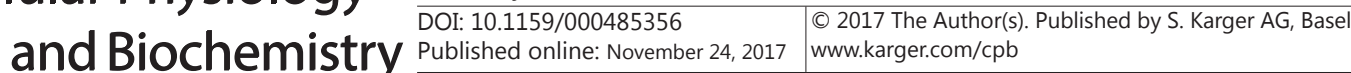

Du et al.: Downregulated MiR-195 Targets SMAD7 in BAV

Alizarin Red S staining to detect calcification

Alizarin Red S staining was performed to detect calcium deposition in the matrix monolayer of cultured VICs. Briefly, transfected cells were treated with TGF- $\beta$ for 14 days and then were washed twice with phosphate-buffered saline, fixed in $4 \%$ paraformaldehyde for 5 minutes at $4{ }^{\circ} \mathrm{C}$ and then were stained with $2 \%$ Alizarin Red S at a pH of 4.2 for a period of 5 minutes at room temperature, following a rinse with distilled water. The remaining calcium-bound Alizarin Red S was extracted by adding $10 \%$ cetylpyridinium chloride for 10 minutes. The determination of the optical density (OD) was carried out at $570 \mathrm{~nm}$ by measuring the absorbance using a spectrophotometer. All the samples were set up in triplicate. Wells at a density of $1.5 \times 10^{5}$ cells/well of untreated porcine VICs without TGF- $\beta$ were used as a control. The data were normalized by the protein content detected in the cellular layer using the Bradford protein assay method.

\section{Gelatin zymography}

Gelatin zymography was used to determine the amount of matrix metalloproteinase (MMP)-2, 9 enzymes produced within the cultured cell monolayers. Briefly, equal amounts protein (equivalent of $10^{5}$ cells) of nonreduced samples were loaded into $10 \%$ zymogram gels. After electrophoresis, the gels were washed with $2.5 \%$ Triton X-100 and were shaken in a renaturing buffer for 30 minutes, followed by incubation in a developing buffer (Life Technologies, Grand Island, NY, USA) at $37^{\circ} \mathrm{C}$ overnight. The gels were then stained in a Coomassie Blue solution for 1 hour before being destained in a 10\% acetic acid/30\% ethanol solution for 1 hour, followed by storage in a $2 \%$ acetic acid solution. The gels were imaged using a ChemiDoc XRS imager (Bio-Rad, Hercules, CA, USA), and the band densities were calculated using the gel analysis tool in ImageJ software.

\section{Statistical Analysis}

Univariate analysis was performed using chi-squared test for categorical variables and Student's t-test for continuous variables. The data are presented as the means \pm standard deviation. A value of $\mathrm{P}<0.05$ (2-sided) was considered to be statistically significant. The data were analyzed using IBM SPSS statistics software (version 19.0).

\section{Results}

\section{BAV and TAV Patient Characteristics}

Information concerning the patients involved in this experiment is listed in Table 1. Overall, $30.0 \%$ of the patients were women, $28.0 \%$ had hypertension, $20.0 \%$ had hypercholesterolemia, and $10.0 \%$ had diabetes mellitus. The BAV group was significantly younger than the DTV group. No significant differences were found in the preoperative cardiac risk factors between the BAV and TAV patients. Preoperative transthoracic echocardiographic measurements, including the left ventricular ejection fraction, aortic valve area, and mean transvalvular gradient, were not different between the BAV and TAV patients. All the selected patients were considered to have severe AS with a mean valve area less than $0.75 \mathrm{~cm}^{2}$. The diameter of the ascending aorta was greater in the BAV patients than in the RTV patients.

\section{Calcified BAV Leaflets Express Lower miR-195 than Calcified TAV Leaflets}

Based on the previous studies and literature, we selected several microRNAs-i.e., miR26a, miR-30b, miR-195 (as osteoblastic differentiation-associated miRs), and miR-486, miR139, miR-192 (as shear-responsive miRs)—that may participate in modifying the progress of early calcification in BAV disease as possible targets to carry out RT-PCR analysis [10, 13, 14]. Compared with rheumatic and degenerative TAV leaflets, the expression of miRNA-195 in BAV leaflets was significantly decreased by 15.0-fold and 10.9-fold, respectively (Fig. 1a), and the RNA level of miRNA-486 in BAV leaflets was decreased by 5.1-fold and 7.6-fold, respectively (Fig. 1d). However, the miR-26a, miR-30b, miR-139, and miR-192 expression levels were unchanged among all the groups (Fig. 1b, 1c, 1e, and 1f). Based on our initial findings, we further investigated the role of miR-195 in this study because it demonstrated the largest fold changes in BAV tissue.

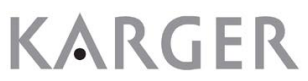




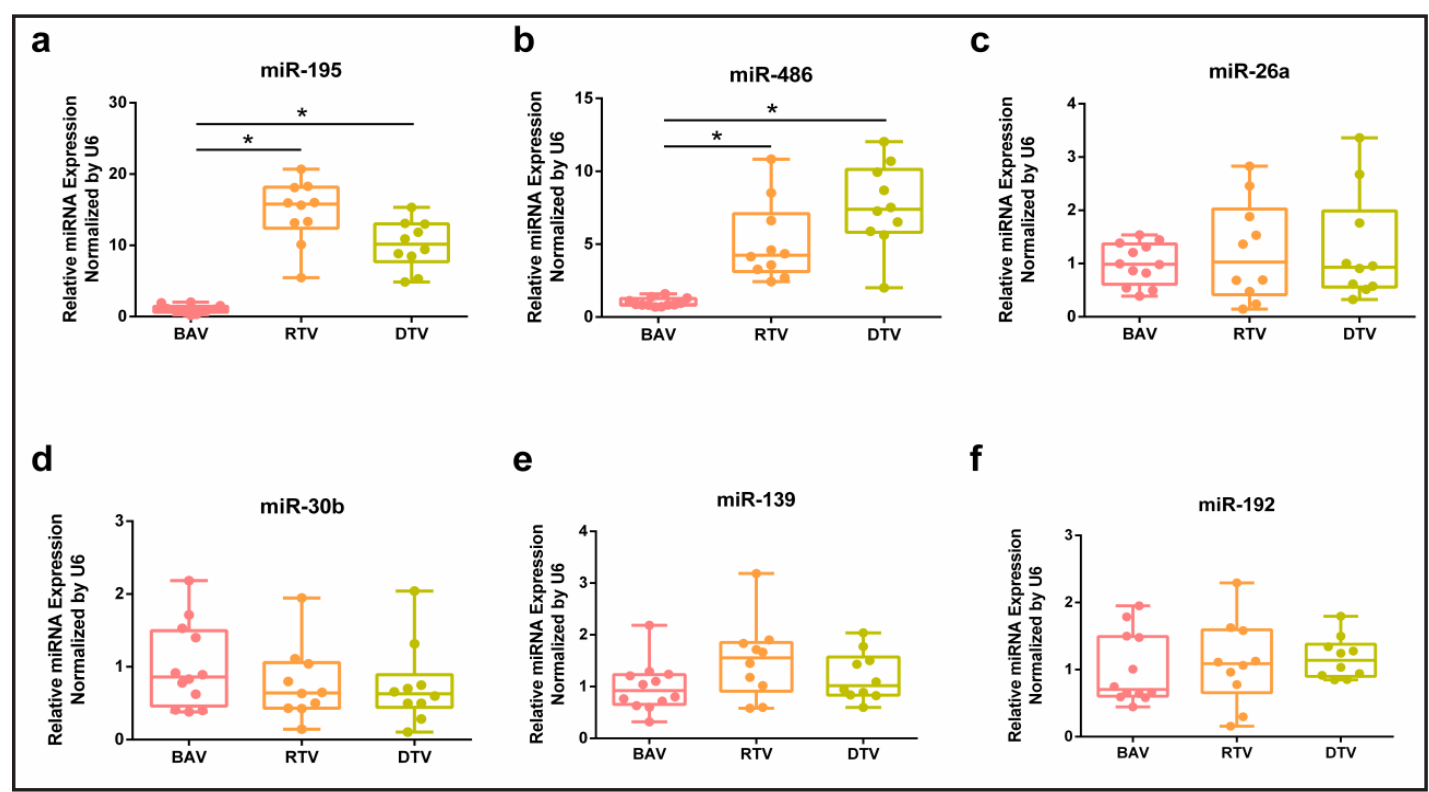

Fig. 1. Calcified BAV Leaflets Express Lower miR-195 and miR-486. (a) Reverse transcription-polymerase chain reaction showed that miR-195 expression was significantly lower in human bicuspid aortic valve (BAV) leaflets than in human rheumatic tricuspid aortic valve (RTV) leaflets and human degenerative tricuspid aortic valve (DTV) leaflets. (b) miR-486 expression was also significantly lower in the BAV group than in the RTV and DTV groups. (c d, e, and f) The expression levels of miR-26a, miR-30b, miRNA-139, and miR-192 showed no significant changes among the three groups. ${ }^{*} \mathrm{P}<0.05$. The data are shown as means \pm SD ( $n=12$ in the BAV group, $n=10$ in the RTV group, $n=10$ in the DTV group).

miR-195 is Negatively Correlated with SMAD7 Levels in BAV Tissues

Using online bioinformatic analysis software (TargetScan Version 7.0 and http:// www.microRNA.org) to predict target genes of miR-195, we found that the calcificationrelated gene SMAD7 is a potential target of miR-195. To test the involvement of miR-195 in the regulation of SMAD7 expression, we first examined SMAD7 mRNA expression in valve tissues by real-time PCR and found that the mRNA expression of SMAD7 in BAV tissues was 1.7-fold and 1.6-fold higher than that in RTV and DTV tissues, respectively (Fig. 2a). Western blotting was performed to examine the SMAD7 protein levels in all three groups. The statistical results showed that the relative density of SMAD7 in BAV tissues was 2.4-fold and 1.5-fold higher than that in RTV and DTV tissues, respectively (Fig. 2b). We also conducted immunohistochemical staining for SMAD7 in all tissue samples, and quantification analysis demonstrated that leaflet tissues from BAV expressed a 1.9-fold and 2.2-fold increase in SMAD7 protein compared with that in RTV and DTV leaflets, respectively (Fig. 2c and $2 \mathrm{~d})$. Pearson's correlation analysis showed that the SMAD7 protein level was correlated negatively with miR-195 ( $R=-0.712, \mathrm{P}<0.01)$ (Fig. 2e).

Validation of SMAD7 as the Direct Target of miR-195

To validate the possibility that miR-195 directly targets $S M A D 7$, we first found the potential binding site for miR-195 according to the bioinformatic analysis. Computational analysis showed that the seed sequence of hsa-miR-195 was completely complementary to the 56-62 nt of 3'UTR in SMAD7 mRNA, and interspecies homology search showed that this site was conserved across several different species of mammals (Fig. 3a). Next, we constructed a pmirGLO-SMAD 3'-UTR-WT vector containing the luciferase gene, the 3'UTR region of SMAD7 and a pmirGLO-SMAD 3'-UTR-MUT vector with a point mutation at the predicted miR-195-binding site (see Fig. 3b). Next, various reporter constructs were transfected into 293T cells together with the miR-195 mimic or miRNA negative control, 
Fig. 2. MiR-195 is negatively correlated with SMAD7 levels in BAV tissues. (a) and (b) The expression of SMAD7 was higher in the human BAV group than in the human RTV and DTV groups at both the mRNA and protein levels. (c) and (d) The representative image of the slices assessed by immunohistochemistry and quantification analysis showed more SMAD7 expression in the BAV group than the RTV

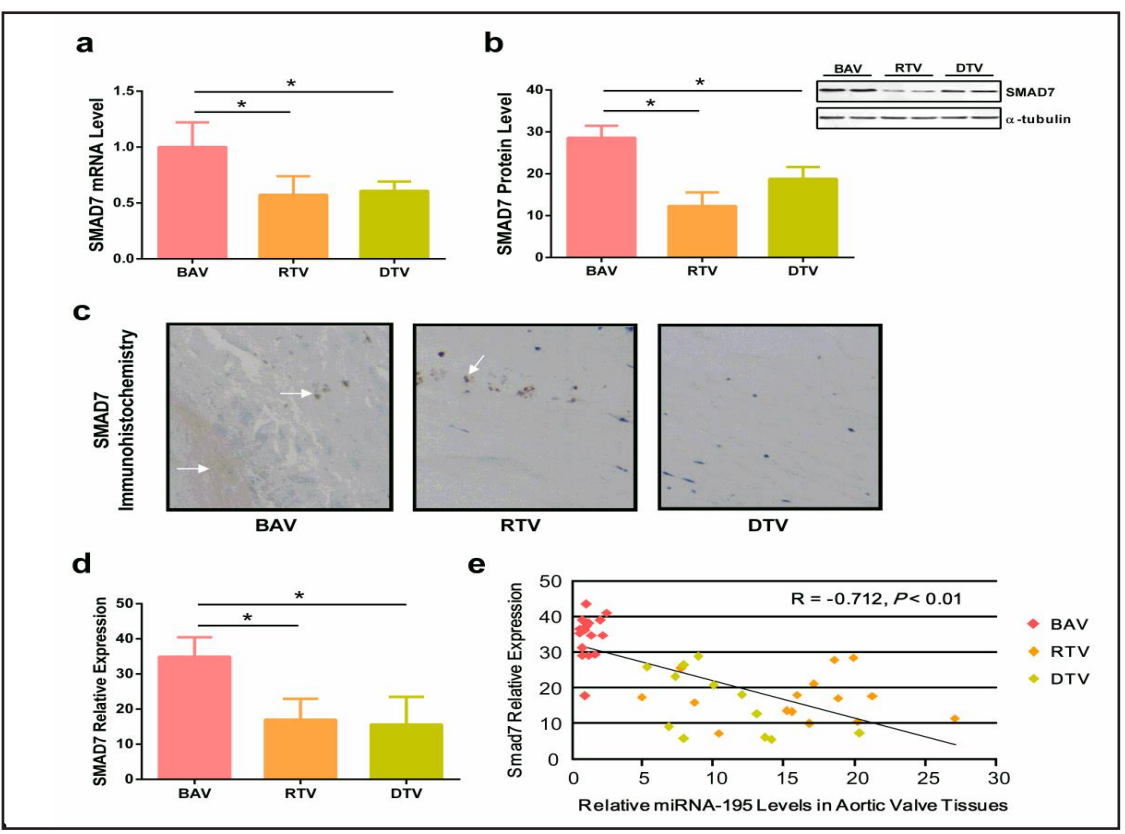
and DTV groups. The arrows indicate tissue with SMAD7-positive staining (brown). (e) Pearson's correlation analysis showed that the SMAD7 protein level was negatively correlated with miR-195 $(\mathrm{R}=-0.712$, $\mathrm{P}<0.01)$. ${ }^{*} \mathrm{P}<0.05$. The data are shown as means \pm SD $(\mathrm{n}=12$ in the BAV group, $\mathrm{n}=10$ in the RTV group, $\mathrm{n}=10$ in the DTV group for SMAD7 mRNA expression experiments; $n=17$ in the BAV group, $n=15$ in the RTV group, $\mathrm{n}=12$ in the DTV group for Pearson's correlation analysis).

and the luciferase activity was analyzed. The miR-195 mimic repressed luciferase activity significantly in the 3'-UTR-WT clones but not in the 3'-UTR-MUT clones. Meanwhile, it did not show any alteration in the cells transfected with empty vectors (Fig. 3c). These results suggest that miR-195 inhibits the expression of SMAD7 by directly binding to the SMAD7 3'UTR in the pmirGLO reporter plasmid and leads to the reduction of luciferase activity.

\section{miR-195 Regulates SMAD7 Expression in VIC Cultures}

We subsequently investigated the functional consequence of the miR-195-SMAD7 mRNA association in porcine VICs in culture. miR-195 was overexpressed by transfecting porcine VICs with the miR-195 mimics or decreased by transfecting cells with a miR-195 inhibitor. By $24 \mathrm{~h}$ after transfection with the miR-195 mimics, the miR-195 levels increased substantially (Fig. 4a). The increased levels of miR-195 lowered the SMAD7 mRNA levels by 2.2-fold (Fig. 4b). On the other hand, the decreased levels of endogenous miR-195 induced by transfecting VICs with the miR-195 inhibitor resulted in increased SMAD7 mRNA levels by 3.5 -fold (Figures $4 \mathrm{c}$ and $4 \mathrm{~d}$ ).

\section{Inhibition of miR-195 Reverses the Progression of Cellular Calcification}

To determine whether the change in miR-195 can affect the progression of calcification in cultured VICs, these transfected cells were investigated under osteogenic conditions by treating them with $10 \mathrm{ng} / \mathrm{mL}$ TGF- $\beta$. Alizarin Red S staining was applied to these cells at day 14 to determine calcium deposition quantitatively. Compared with cells in normal medium, the addition of TGF- $\beta$ induced 3.9-fold and 3.7-fold increases in calcification in the negative control and miR-195 mimic-transfected VICs, respectively. Interestingly, a significant further increase (1.62-fold) was detected in mi-R 195-repressing VICs compared with the negative control with TGF- $\beta$ treatment (Fig. 5 a and $5 b$ ).

To investigate the potential mechanism of the promotion of VIC calcification by suppressing miR-195, the BMP-2 signaling pathway was studied in these cells under 
Fig. 3. Validation of SMAD7 as the Direct Target of miR-195. (a) Predicted binding site of miR-195 with SMAD7. Interspecies homology search showed that this site was conserved across several different species of mammals. (b) The seed sequence of miR-195 was complementary to the 56-62 nt of the 3'UTR in SMAD7 mRNA. The mutant construct (pmirGLO-UTRMUT) carries a point mutation-i.e., a cytosine was replaced by a guanine in the 3'-UTR segments of human SMAD7, as indicated with an asterisk in the Fig.. (c) The miR-195 mimic repressed luciferase activity significantly in the 3'-UTR-WT clones but not in the 3'-UTR-MUT clones or cells transfected with empty vectors. $* \mathrm{P}<0.05$ for the indicated values versus the vector-only group. The data are shown as means \pm SD. Experiments were performed in triplicate and were independently repeated three times.

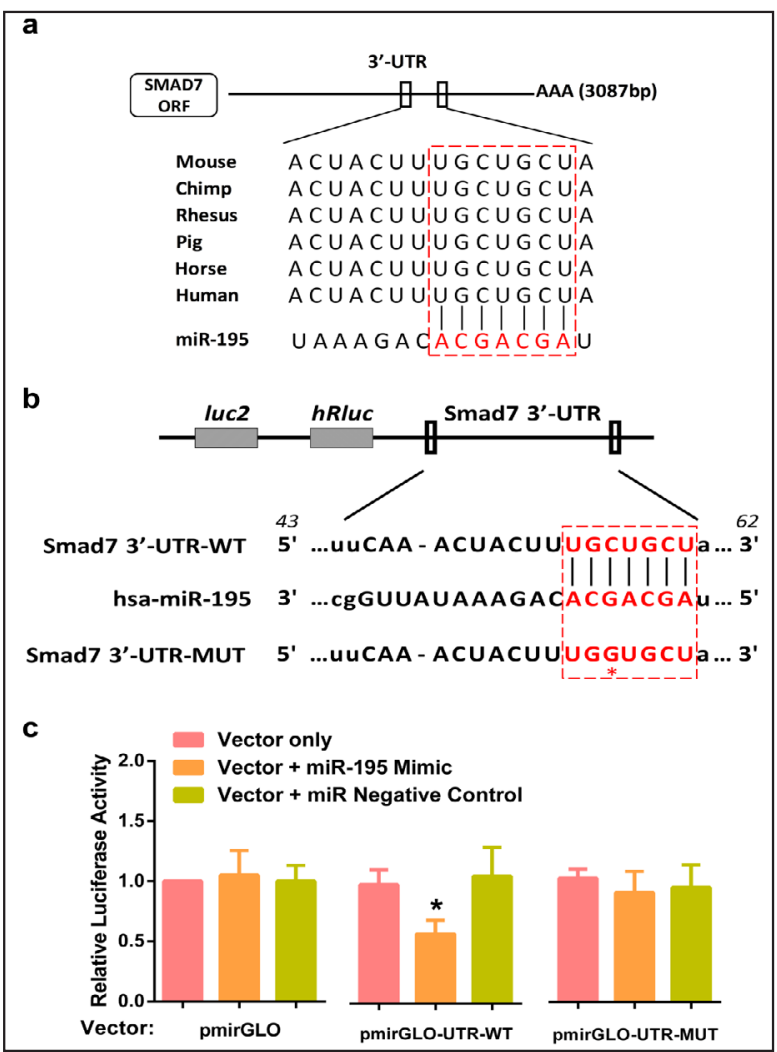

Fig. 4. miR-195 Regulates SMAD7 expression in VIC Cultures. (a) and (b) In cultured valvular interstitial cells, miR-195 mimic treatment increased SMAD7 mRNA expression. (c) and (d) In cultured valvular interstitial cells, miR-195 inhibitor treatment blocked the mRNA expression of SMAD7. * $\mathrm{P}<0.05$ for the indicated values versus the negative control group. The data are shown as means \pm SD. Experiments were performed in triplicate and were independently re-

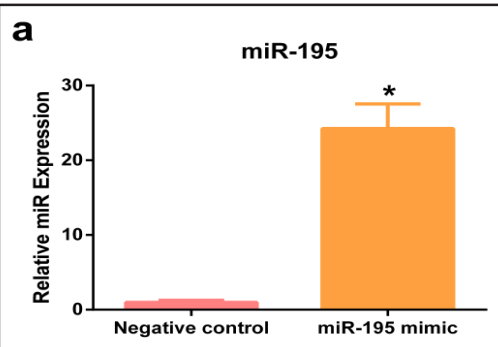
b SMAD7

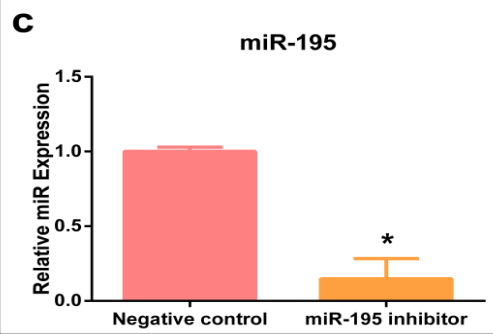

d
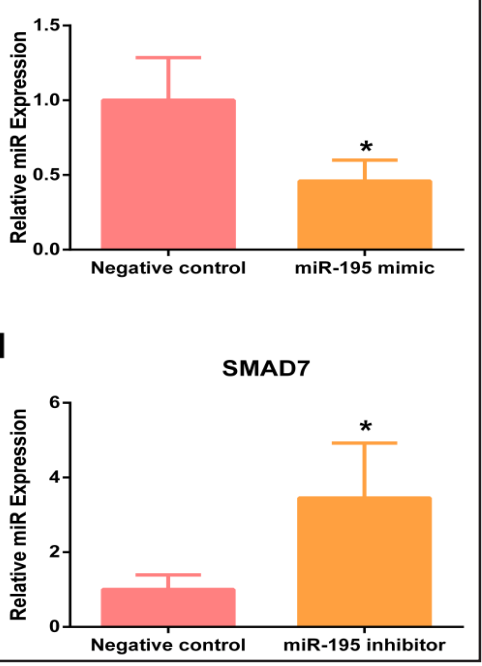
peated three times.

osteogenic conditions by treating them with TGF- $\beta$. The level of transcripts of $B M P-2$ in miR195 mimic-transfected VICs was similar to that in control cells, while a significant increase was seen in miR-195 inhibitor-transfected cells (Fig. 5c). Similar patterns of change were found in the transcript levels of the downstream pro-osteogenic transcription factor RunX2 in these cultured VICs (Fig. 5d). Furthermore, increased RunX2 transcription levels were confirmed at the protein level after TGF- $\beta$ treatment in miR-195-repressing VICs (Fig. 5e).

Furthermore, the activities of MMP-2 and MMP-9 enzymes among these VICs were examined after 14 days of TGF- $\beta$ treatment because the extracellular matrix (ECM) remodeling KARGER 
Fig. 5. Inhibition of miR195 reverses the progression of cellular calcification in VIC Cultures. (a) and (b) Representative images and quantification of Alizarin Red S staining for calcium deposition in cultured VICs with and without TGF- $\beta$ treatment. *, $\mathrm{P}<0.05$ versus the negative control group without TGF- $\beta$ treatment. **, $\mathrm{P}<0.05$ versus the miR195 mimic group without TGF- $\beta$ treatment. ***, $\mathrm{P}<0.05$ versus the negative control group with TGF- $\beta$ treatment. (c) The level of transcripts of BMP-2 in cultured VICs under osteogenic conditions. (d) The transcript levels of RunX2 in the cultured VICs under osteogenic conditions. (e) Western blot imaging and quantification of the protein levels of RunX2 in these cells after TGF- $\beta$

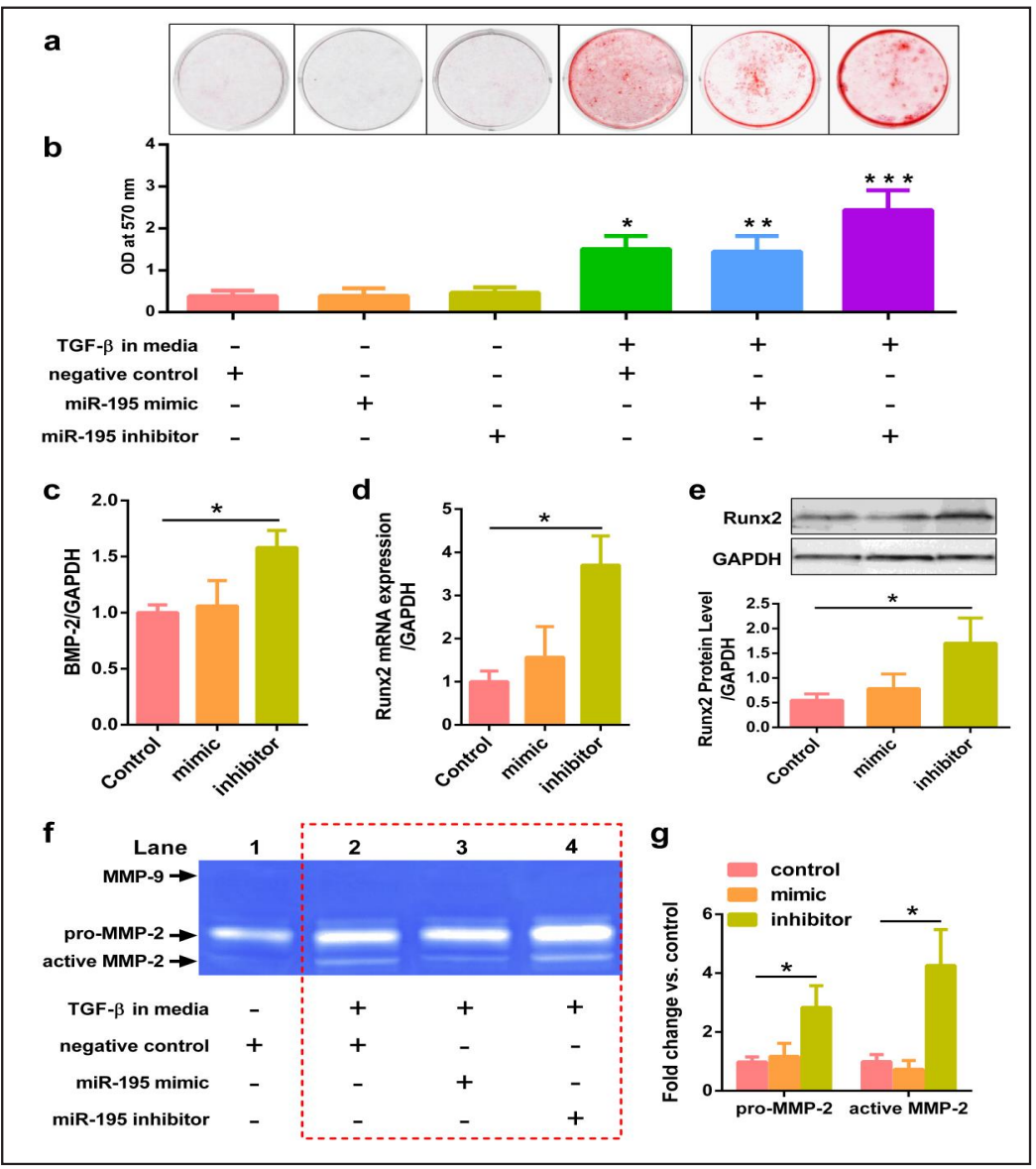
treatment. (f) Representative gel image of gelatin zymography for MMP-2 and -9 expression in cultured VICs with and without TGF- $\beta$ treatment. (g) Image analysis of the bands (proMMP-2 and active MMP-2) for cultured cells under osteogenic conditions. ${ }^{*} \mathrm{P}<0.05$. The data are shown as means \pm SD. Experiments were performed in triplicate and were independently repeated three times.

and abnormal activation of MMPs (a family of matrix enzymes that cleave various ECM components) are the early markers of VIC activation and calcification. Gelatin zymography showed that VICs in both regular and osteogenic media expressed inactive (pro-) MMP-2; however, activated MMP-2 was observed only in VICs under osteogenic conditions (Fig. 5f, lane 2, 3, and 4). The bands for MMP-9 were not detectable in these cell culture studies. Quantitative analysis of the images showed that, following TGF- $\beta$ treatment, miR-195 mimic transfection had no effect on the activities of either pro-MMP-2 or active MMP-2 compared with control cells, while miR-195-repressing VICs demonstrated a significantly increased amount of both inactive and active MMP-2 compared with the control VICs (Fig. 5g).

\section{BAV Leaflets Present More Osteogenic Alteration towards Calcification}

We finally examined the functional alteration associated with cellular calcification in valve tissues. First, Von Kossa staining demonstrated similar calcium deposits among BAV, RTV and DTV leaflet slices (Fig. 6a). Immunohistochemistry for MMP-2 and MMP-9 revealed that leaflet tissues from BAV expressed a significant increase in MMP-2 and MMP-9 protein compared with RTV and DTV leaflets according to image quantification analysis (Fig. 6b and 6c). Finally, Sirius Red staining revealed more positivity areas in BAV than in RTV and DTV tissues (Fig. 6d). 
Fig. 6. BAV Leaflets present more functional alteration associated with calcification. (a) Representative images and quantification of Von Kossa staining for the deposit of calcium in human aortic valve leaflets. (b) and (c) Representative images and quantification of immunohistochemical staining of matrix metalloprotein-2 (MMP2) and matrix metalloprotein-9 (MMP9) expression in human aortic valve leaflets. (d) Representative images and quantification of Sirius Red staining of collagen in human aortic valve leaflets. $* \mathrm{P}<0.05$. The data are shown as means \pm SD $(n=10$ in the BAV group, $n=10$ in
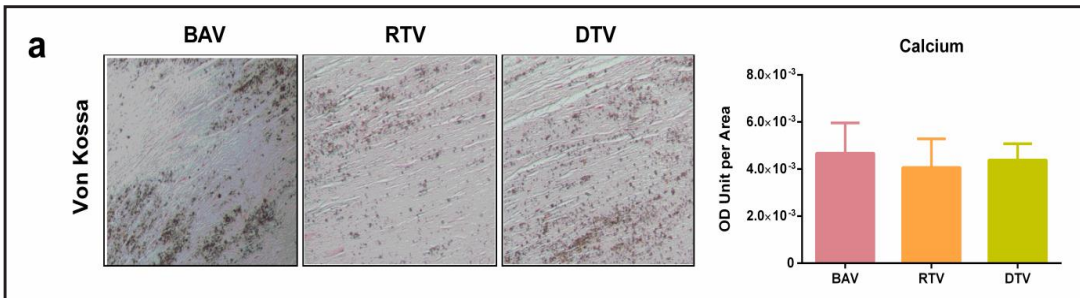

b
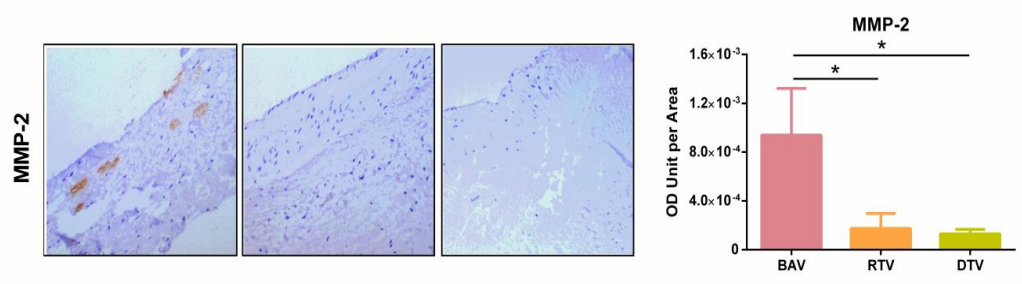

C
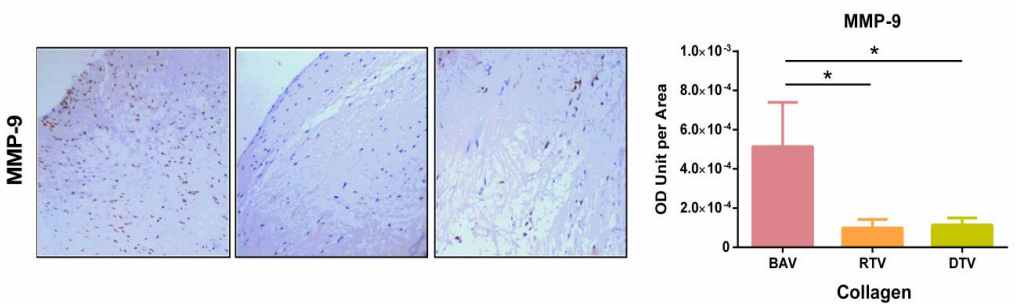

d
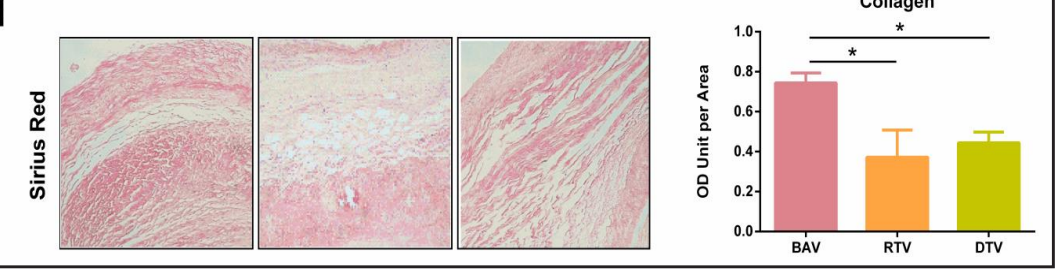

the RTV group, $n=10$ in

the DTV group).

\section{Discussion}

In the present report, we tested some promising microRNAs by RT-PCR, demonstrating representative downregulation of miR-195 in BAV leaflets compared with that in TAV leaflets. We selected both rheumatic TAVs and degenerative TAVs as control groups to study the difference between BAV and TAV. The comparisons revealed significant distinction in miR-195, suggesting that this microRNA expresses differentially in BAV and TAV regardless of other pro-calcific influences. Meanwhile, a higher mRNA expression of SMAD7 in BAV leaflets was observed. Next, we found that the protein expression of SMAD7 was consistent with the alteration of the corresponding mRNA. Furthermore, we demonstrated that the downregulation of miR-195 in cultured porcine VICs facilitated the mRNA expression of SMAD7. miR-195 is an important member of the micro-15/16/195/424/497 family, which is activated in multiple diseases, such as cancer, heart failure, and schizophrenia [15]. SMAD7 is an important mediator belonging to the inhibitory SMADs that play important roles especially in the SMAD-dependent signaling [16]. However, to our knowledge, the miR195 differential expression between human BAV and TAV leaflet and its action mechanism related to SMAD7 in the early calcification of bicuspid aortic valves have not been shown before, which is the novelty of this article.

Several lines of evidence have suggested that miR-195 is an essential regulator in valve calcification. Vishal Nigam et al. noted that miR-195 levels were reduced by 59\% in stenotic samples by qRT-PCR, and miR-195-treated AVICs showed increased mRNA levels of calcification-related genes such as BMP2 and RUNX2 [13]. The stenotic valve is more 
common within the bicuspid aortic valves, supporting our finding that miR-195 has lower expression in BAV leaflets.

There are also several previous studies from other groups supporting our results concerning the relationship between miR-195 and SMAD7. Chen G et al. noted that. in colon cells, the antagonism of miR-195 enhanced SMAD7 translation, and luciferase reporter assay in the Caco- 2 cell line revealed a repressive effect of miR-195 via a single SMAD7 3'-UTR target site [17]. The Duan Yingjun group found that, in the human glioblastoma multiform cell line and human glioma cancer tissues, miR-195 targeted and inhibited SMAD7 expression [18]. The Guo D group indicated that miR-195 inhibited MTEC1 proliferation, at least in part, via the downregulation of SMAD7 in mouse medullary thymic epithelial cells [19]. However, in the article by Nigam and colleagues [13], the overexpression of miR-195 in human aortic valve interstitial cells leads to the upregulation of SMAD7, a finding that is opposite to what we and others found in BAV patients and other mammalian cell types. Different seed region positions between species could explain the different observations. However, the seed region is conservative among many mammalian species, including human and pigs, as shown in Fig. 3. In our study, we clearly demonstrated the inverse correlation of miR-195 and SMAD7 both in human samples and pig cells at the mRNA and protein levels. We also noticed that, in Nigam's paper, the human aortic VICs were from recipient hearts at the time of cardiac transplantation. These patients were subjected to chronic and advanced pathological and medical conditions that might have a complicated impact on the VICs in their aortic valves. It could explain why the miR-195-SMAD relationship and individual expression levels in their VICs are different from those in the present study in which the VICs were harvested from healthy pigs.

Furthermore, some evidence provided by published articles was also consistent with our point of view that SMAD7 signaling, the crucial step in our study, plays a key role in the early calcification of BAV. The MohanKumar K group proposed that, in human necrotizing enterocolitis macrophages, increased SMAD7 expression augmented nuclear factor-kappa $\mathrm{B}(\mathrm{NF}-\kappa \mathrm{B})$ activation and cytokine production via increasing the expression of IKK- $\alpha$ [20]. Zorzi F et al. and Ardizzone $S$ et al. both illustrated that, in human inflammatory bowel tissue, interrupting SMAD7 inhibits the inflammation action [21, 22].

Examination of human calcified aortic valve tissue and in vitro experiments using AVICs have demonstrated that there are several gene pathways involved in calcification including TGF-Beta, BMP2, NOTCH1, and SMADs [23-25]. To determine the relationship between SMAD7 and aortic calcification, we have focused on several previous studies. Miyazawa K et al. and Kim CR et al. proposed that SMAD7 inhibits both transforming growth factor â (TGF- $\beta$ )and BMP-induced SMAD signaling $[26,27]$. These studies related to SMAD7 and TGF- $\beta$ have suggested that SMAD7 ultimately promotes calcification through interrupting TGF- $\beta$ signaling. All the above findings indicate that the miR-195 regulation of SMAD7-dependent calcification might underlie the altered susceptibility toward the early calcification observed in patients with BAV.

Finally, the functional change between BAVs and TAVs was checked in the patients' valve leaflets. We noticed that, although the two types of leaflets presented a similar degree of calcification, the MMP2 protein, MMP9 protein and collagen expression were all expressed at a higher level in BAV leaflets, indicating a disorder in the matrix of BAV leaflets. Thus, we propose the hypothesis that, in BAV leaflets, the downregulation of miR195 enhances SMAD7 signaling, further promoting the fibrosis in extracellular matrix and causing valvular calcification. Some studies from other laboratories have supported our point of view. The Zampetaki A group reported that, in mice treated with antagomiR-195, higher aortic elastin expression was associated with an increase in MMP-2 and MMP-9 [28]. The Pardali E. group stated that TGF $\beta$ binding proteins are activated by plasmin, reactive oxygen species, acidic microenvironment, matrix metalloproteinases (MMP-2 and -9) and $\alpha 6$ integrin [29]. However, different results in similar studies also exist. The Guanxian Liu group proposed that SMAD7 disrupts thrombospondin-enhanced NF- $\kappa B$ signaling in SMAD7 knockout mice in their renal tissue, which seems to be contrary to our point that SMAD7 
contributes to calcification through NF-kB-BMP2 signaling [30]. However, we performed immunohistochemical experiments and some staining tests using slides from human aortic valve leaflets, while they carried out research using different tissues from different species; thus, no conflict exists.

Our study possesses limitations. Initially, based on previous studies probing the noncoding RNA action in cardiovascular calcification $[13,14]$, we selected several microRNAs (including miR-26a, miR-30b, miR-195, miR-486, miR-192, and miR-139) as potential targets, and then checked their RNA expression in bicuspid leaflets and tricuspid leaflets to confirm miR-195 as the first step of our study. We admit that the results should have been more solid if we had performed a microRNA array on the human aortic valves we obtained. Moreover, our present study could not elucidate the downstream of facilitated SMAD7 signaling, and whether the NF- $\kappa$ B-BMP2-Runx2 pathway works in the bicuspid valve in the specific human tissue remains to be explored more comprehensively.

\section{Conclusion}

Our study has demonstrated that miR-195 is downregulated more in stenotic aortic leaflets from BAV patients compared with that from patients with TAV. We have also shown that the downregulation of miR-195 is associated with valvular calcification via targeting $S M A D 7$, which involves the progress of fibrosis and remodeling of the extracellular matrix. Our findings provide new clues regarding the mechanism of accelerated valvular calcification in BAV patients.

\section{Acknowledgements}

This work was supported, in part, by the Priority Academic Program of Jiangsu Higher Education Institutions (Grants JX10231801) and the Natural Science Foundation of Jiangsu Province (BK20151590). The authors confirm that the funders had no influence over the study design, content of the article, or selection of this journal.

\section{Disclosure Statement}

No potential conflicts of interest exist with any companies/organizations whose products or services may be discussed in this manuscript.

\section{References}

$\rightarrow 1$ Siu SC, Silversides CK: Bicuspid Aortic Valve Disease. JACC 2010;55:2789-2800.

-2 Girdauskas E, Borger MA: Bicuspid aortic valve and associated aortopathy: an update. Semin Thorac Cardiovasc Surg 2013;25:310-316.

-3 Wirrig EE, Yutzey KE: Conserved transcriptional regulatory mechanisms in aortic valve development and disease. Arterioscler Thromb Vasc Biol 2014;34:737-741.

-4 Farrar EJ, Pramil V, Richards JM, Mosher CZ, Butcher JT: Valve interstitial cell tensional homeostasis directs calcification and extracellular matrix remodeling processes via RhoA signaling. Biomaterials 2016;105:2537.

5 Wang Y, Wu, B, Farrar, E, Lui, W, Lu P, Zhang, D, Alfieri CM, Mao K, Chu, M, Yang D, Xu D, Rauchman M, Taylor V, Conway SJ, Yutzey KE, Butcher JT, Zhou, B: Notch-Tnf signalling is required for development and homeostasis of arterial valves. Eur Heart J 2015;38:675-686

-6 Mohler ER 3rd, Gannon F, Reynolds C, Zimmerman R, Keane MG, Kaplan FS: Bone formation and inflammation in cardiac valves. Circulation 2001;103:1522-1528. 


\section{Cellular Physiology Cell Physiol Biochem 2017;44:884-896

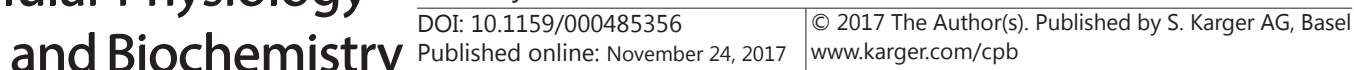 and Biochemistry Published online. November 24,2017 mir. wark SMAD7 in BAV}

7 Kaden JJ, Bickelhaupt S, Grobholz R, Vahl CF, Hagl S, Brueckmann M, Haase KK, Dempfle CE, Borggrefe M: Expression of bone sialoprotein and bone morphogenetic protein-2 in calcific aortic stenosis. J Heart Valve Dis 2004;13:560-566.

8 Leopold, JA: MicroRNAs Regulate Vascular Medial Calcification. Cells 2014;3:963-980.

-9 Oury, C, Servais, L, Bouznad N, Hego A, Nchimi A, Lancellotti P: MicroRNAs in Valvular Heart Diseases: Potential Role as Markers and Actors of Valvular and Cardiac Remodeling. Int J Mol Sci 2016;17:1120.

10 Rathan S, Ankeny CJ, Arjunon S, Ferdous Z, Kumar S, Fernandez EJ, Heath JM, Nerem RM, Yoganathan AP, Jo $\mathrm{H}$ : Identification of side- and shear-dependent microRNAs regulating porcine aortic valve pathogenesis. Sci Rep 2016;6:25397.

-11 Patel, V, Carrion K, Hollands A, Hinton A, Gallegos T, Dyo J, Sasik R, Leire E, Hardiman G, Mohamed SA, Nigam S, King CC, Nizet V, Nigam V: The stretch responsive microRNA miR-148a-3p is a novel repressor of IKBKB, NF- B signaling, and inflammatory gene expression in human aortic valve cells. FASEB J 2015;29:1859-1868.

12 Wang Y, Chen S, Deng C, Li F, Wang Y, Hu X, Shi F, Dong N: MicroRNA-204 Targets Runx2 to Attenuate BMP2-induced Osteoblast Differentiation of Human Aortic Valve Interstitial Cells. J Cardiovasc Pharmacol 2015;66:63-71.

13 Nigam, V, Sievers HH, Jensen BC, Sier HA, Simpson PC, Srivastava D, Mohamed SA: Altered microRNAs in bicuspid aortic valve: a comparison between stenotic and insufficient valves. J Heart Valve Dis 2010;19:459-465.

14 Holliday CJ, Ankeny RF, Jo H, Nerem RM: Discovery of shear- and side-specific mRNAs and miRNAs in human aortic valvular endothelial cells. Am J Physiol Heart Circ Physiol 2011;301:H856-867.

15 He JF, Luo YM, Wan XH, Jiang D: Biogenesis of MiRNA-195 and its role in biogenesis, the cell cycle, and apoptosis. J Biochem Mol Toxicol 2011;25:404-408.

-16 Li Q: Inhibitory SMADs: potential regulators of ovarian function. Biol Reprod 2015;92:950.

17 Chen G, Cao S, Liu F, Liu Y: miR-195 plays a role in steroid resistance of ulcerative colitis by targeting Smad7 Biochem J 2015;471:357-367.

18 Duan Y, Chen Q: TGF-beta1 regulating miR-205/miR-195 expression affects the TGF-beta signal pathway by respectively targeting SMAD2/SMAD7. Oncol Rep 2016;36:1837-1844.

19 Guo D, Ye Y, Qi J, Xu L, Zhang L, Tan X, Tan Z, Yu X, Zhang Y, Ma Y, Li Y: MicroRNA-195a-5p inhibits mouse medullary thymic epithelial cells proliferation by directly targeting Smad7. Acta Biochim Biophys Sin (Shanghai) 2016;48:290-297.

20 MohanKumar K, Namachivayam K, Chapalamadugu KC, Garzon SA, Premkumar MH, Tipparaju SM, Maheshwari A: Smad7 interrupts TGF-beta signaling in intestinal macrophages and promotes inflammatory activation of these cells during necrotizing enterocolitis. Pediatr Res 2016;79:951-961.

21 Zorzi F, Angelucci E, Sedda S, Pallone F, Monteleone G: Smad7 antisense oligonucleotide-based therapy for inflammatory bowel diseases. Dig Liver Dis 2013;45:552-555.

22 Ardizzone S, Bevivino G, Monteleone G: Mongersen, an oral Smad7 antisense oligonucleotide, in patients with active Crohn's disease. Therap Adv Gastroenterol 2016;9:527-532.

-23 Wu M, Chen G, Li YP: TGF- $\beta$ and BMP signaling in osteoblast, skeletal development, and bone formation, homeostasis and disease. Bone Research 2016;4:16009.

-24 Garg V: Molecular genetics of aortic valve disease. Curr Opin Cardiol 2006;21:180-184.

25 Kurabayashi M: Vascular Calcification - Pathological Mechanism and Clinical Application - Role of vascular smooth muscle cells in vascular calcification. Clin Calcium 2015;25:661-669.

-26 Miyazawa K, Miyazono K, Regulation of TGF-beta Family Signaling by Inhibitory Smads. Cold Spring Harb Perspect Biol 2017;9 DOI: 10.1101/cshperspect.a022095

27 Kim CR, Kim YM, Lee MK, Kim IH, Choi YH, Nam TJ: Pyropia yezoensis peptide promotes collagen synthesis by activating the TGF-beta/Smad signaling pathway in the human dermal fibroblast cell line Hs27. Int J Mol Med 2017;39:31-38

28 Zampetaki A, Attia R, Mayr U, Gomes RS, Phinikaridou A, Yin X, Langley SR, Willeit P, Lu R, Fanshawe B, Fava M, Barallobre-Barreiro J, Molenaar C, So, PW, Abbas A, Jahangiri M, Waltham M, Botnar R, Smith A, Mayr M: Role of miR-195 in aortic aneurysmal disease. Circ Res 2014;115:857-866.

29 Pardali E, Ten DP: TGFbeta signaling and cardiovascular diseases. Int J Biol Sci 2012;8:195-213.

-30 Liu GX, Li YQ, Huang XR, Wei LH, Zhang Y, Feng M, Meng XM, Chen HY, Shi YJ, Lan HY: Smad7 inhibits AngIImediated hypertensive nephropathy in a mouse model of hypertension. Clin Sci (Lond) 2014;127:195-208. 